\title{
The X-ray spectra of the flaring and quiescent states of AT Microscopii observed by XMM-Newton ${ }^{\star}$
}

\author{
A. J. J. Raassen ${ }^{1,2}$, R. Mewe ${ }^{1}$, M. Audard ${ }^{3}$, and M. Güdel ${ }^{4}$ \\ 1 SRON National Institute for Space Research, Sorbonnelaan 2, 3584 CA Utrecht, The Netherlands \\ 2 Astronomical Institute "Anton Pannekoek", Kruislaan 403, 1098 SJ Amsterdam, The Netherlands \\ 3 Columbia Astrophysics Laboratory, Mail code 5247, 550 West 120th Street, New York, NY 10027, USA \\ ${ }^{4}$ Paul Scherrer Institut, Würenlingen \& Villigen, 5232 Villigen PSI, Switzerland
}

Received 3 February 2003 / Accepted 29 August 2003

\begin{abstract}
The X-ray spectrum of the late-type M-dwarf binary AT Mic (dM4.5e+dM4.5e) is observed in the wavelength range 1-40 A by means of RGS and EPIC-MOs on board XMM-Newton. During the exposure a flare occured. We have performed a 3-temperature fit and a DEM-modeling to the flaring and quiescent part of the spectrum. We report the coronal temperature distribution, emission measures, and abundances of the flaring and quiescent state of this bright X-ray source. The temperature range stretches from about 1 to $60 \mathrm{MK}$. The total volume emission measure in this temperature interval is $\sim 12.2 \times 10^{51} \mathrm{~cm}^{-3}$ for the quiescent state and $\sim 19.5 \times 10^{51} \mathrm{~cm}^{-3}$ for the flare state. This difference is due to the contribution of the hot temperature component. The high-resolution spectrum of AT Mic, obtained by RGS, is dominated by the H-and He-like transitions of C, N, $\mathrm{O}$, and $\mathrm{Ne}$ and by Fe XVII lines, produced by the plasma with temperatures from 1 to $10 \mathrm{MK}$. The EPIC-MOS spectrum below $10 \AA$ shows $\mathrm{H}$ - and He-like Ne, Si and the iron K-shell transitions. They are produced by the hot component (30 MK). The iron K-shell is more prominent in the flare state. The abundance pattern in the quiescent state of AT Mic shows the depletion of low-FIP elements relative to high-FIP elements, indicating the presence of an I(nverse)FIP effect in this active star. In the flare state, however, some flattening of this IFIP effect is present.
\end{abstract}

Key words. stars: individual: AT Mic - stars: coronae - stars: late-type - missions: - XMM-Newton

\section{Introduction}

Hot outer atmospheres (coronae) are very common for relatively cool stars in the spectral classes F-M. Many of these coronae are characterized by temperatures up to about $20 \mathrm{MK}$ and densities $n_{\mathrm{e}} \gtrsim 10^{10} \mathrm{~cm}^{-3}$. It was already known that the coronae of many stars are different from the solar corona (e.g., reviews by Pallavicini 1989; Mewe 1991). The heating mechanism of these coronae, however, is still not well understood. A variety of possible explanations is present, such as, convection zones with MHD waves transfering energy from the photosphere into the corona, large flares heating plasma by reconnection, or a large number of continuously heating microand nanoflares. High-resolution spectroscopy of stellar systems available from Chandra (Brinkman et al. 2000; Canizares et al. 2000) and XMM-Newton (Brinkman et al. 2001) offers the possibility to study the coronal spectra in great detail and to determine various coronal quantities, such as densities, temperatures, abundances, emission measures, and line ratios, during the flaring states as well as during the quiescent state.

Send offprint requests to: A. J. J. Raassen, e-mail: a.j.j.raassen@sron.nl

* Based on observations obtained with XMM-Newton, an ESA scienc mission with instruments and contributions directly funded by ESA Member States and the USA (NASA).
Late-type stars ( $\mathrm{M}$ and $\mathrm{K}$ dwarfs) show more magnetic activity and higher coronal temperatures than the Sun, characterized by flaring (Pettersen 1989) in the optical, UV, X-ray and radio wavelengths (Pallavicini et al. 1990). To understand the mechanisms that underly the high temperatures and the variety of densities, emission measures, and abundance values observed in coronal systems, the X-ray spectra of stars of type F-M are investigated, especially focused on stars with flare activity. In very active stars, however, the "quiescent state" might be produced by a superposition of flare decays and microflares rather than by a real quiescent coronal plasma. Here we present the investigation of the active M-type binary star AT Mic (dM4.5+dM4.5) at a distance of 10.22 pc (Perryman et al. 1997). Both components are known to flare frequently (Joy \& Wilson 1949). Large optical flares were observed by García-Alvarez et al. (2002), while X-ray flares were studied e.g., by HEAO-1 (Kahn et al. 1979) and by EXOSAT (Pallavicini et al. 1990). The quiescent corona was observed by EUVE (Monsignori Fossi et al. 1995).

\section{Observations}

The X-ray spectrum of AT Mic was observed by RGS and EPIC-MOS on board XMM-Newton on 14 October 2000 
Table 1. Observation log of the data of AT Mic.

\begin{tabular}{lllllr}
\hline \hline Instrument & Filter & Mode & Date-obs-start & Date-obs-end & Performed duration (s) \\
\hline MOS1 & Medium & Small Window & 2000-10-16T00:27:48 & 2000-10-16T07:34:02 & 25394 \\
MOS2 & Medium & Timing & 2000-10-16T00:38:15 & 2000-10-16T07:34:29 & 24794 \\
pn & Medium & Small Window & 2000-10-16T00:41:50 & 2000-10-16T07:41:13 & 25103 \\
RGS1 & None & Spec+Q & 2000-10-16T00:19:12 & 2000-10-16T08:11:48 & 28254 \\
RGS2 & None & Spec+Q & 2000-10-16T00:19:12 & 2000-10-16T08:11:48 & 28254 \\
\hline
\end{tabular}

during revolution 156 . The observation $\log$ of the data is presented in Table 1.

The data were processed by means of the XMM-Newton SAS version 5.3.3 (June 2002). The exposure time was $28.3 \mathrm{ks}$ for RGS and $25.4 \mathrm{ks}$ for EPIC-MOS1, of which $19.0 \mathrm{ks}$ were free of solar flare proton "pollution" applying the criterion that the latter's count rate should be $\lesssim 0.35 \mathrm{c} / \mathrm{s}$. For RGS the first-order net spectra were extracted by including $95 \%$ of the cross dispersion PSF (xpsfincl = 95 in rgsproc) and the background spectra were extracted by excluding $98 \%$ (xpsfexcl $=98$ in rgsproc).

The RGS spectral resolution is $\Delta \lambda \sim 0.07 \AA$ (FWHM), with a maximum effective area of about $140 \mathrm{~cm}^{2}$ around $15 \AA$. The wavelength uncertainty is $7-8 \mathrm{~m} \AA$. The total RGS spectrum runs from 5 to $37 \AA$. Only the range from 8 to $37 \AA$ was used. For EPIC-MOS1, which observed in the small-window mode, we extracted the spectrum by means of a circle centered around the source with a radius of 40 arcsec. The background was obtained by applying the same circle in a source free area at another CCD. The same SAS version was used to construct response matrix and ancillary response files. The range from 1 to $14 \AA$ was used. EPIC-Mos 2 was operating in the timing mode. For more instrumental details on RGS and EPIC-MOS see den Herder et al. (2001) and Turner et al. (2001), respectively. The pn data were used to produce a lightcurve only.

\section{Analysis}

\subsection{Quiescence versus flaring state}

Based on the lightcurves obtained with RGS, EPIC-MOS and pn (see Fig. 1) we have concluded that AT Mic was flaring several times during our observations. A period of about $2.9 \mathrm{ks}$ related to the most prominent flare was extracted from the GTI table as flare state, leaving a pseudo-quiescent part of $14.1 \mathrm{ks}$. Three other weak flares can be seen in Fig. 1. However, they do not dominate and have therefore not been flagged as flares in the further spectral analysis. From Fig. 1 we notice that the top of the flare is not constant, but shows small variations, which seem to be periodic. However, these time intervals are too short to allow for individual spectral analysis.

The observed spectra are shown in Fig. 2 for the quiescent (top) and flaring (bottom) state. As can be seen from Fig. 2, EPIC-MOS has a low resolution, but a high sensitivity compared to RGS. Due to the short exposure time of the flaring state the signal/noise ratio is lower for that spectrum. For both states shown in Fig. 2 it is clear that the X-ray spectrum obtained by RGS in the wavelength range from 5 to $37 \AA$ is

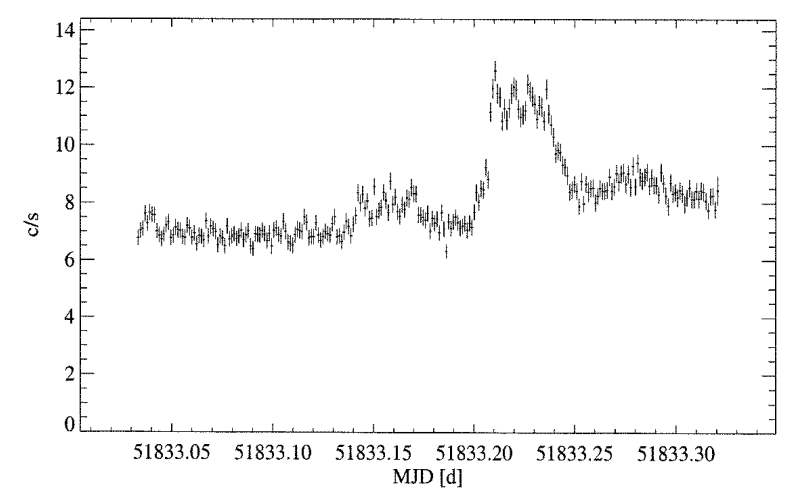

Fig. 1. Lightcurve of AT Mic, observed with pn.

dominated by $\mathrm{H}-$ and He-like transitions of $\mathrm{C}, \mathrm{N}, \mathrm{O}$, and $\mathrm{Ne}$ and by Fe XVII lines, while the low-resolution EPIC-MOS spectrum shows the Fe K-shell transitions around $1.9 \AA$ and the $\mathrm{H}$ and He-like transitions of $\mathrm{Ne}$ and $\mathrm{Si}$. The Fe K-shell feature (see also Table 4) as well as the continuum appear more prominent in the spectrum of the flaring state (bottom panel) than in that of the quiescent state.

\subsubsection{Multi-temperature fitting}

The data of the quiescent state and the flaring state have been fitted with a 3-T Collisional Ionization Equilibrium model using the SPEX code (Kaastra et al. 1996a) in combination with an updated version of MEKAL (Mewe et al. 1995; Phillips et al. 1999). The MEKAL database is publicly available ${ }^{1}$ as an extended list of fluxes of more than 5400 spectral lines. Figure 3 shows the comparison of the quiescent spectrum with the bestfit model. The fits have been performed by attaching the errors to the data ("fit weight data"), given in the columns "data", and by attaching the errors to the model ("fit weight model"), given in the columns "model". In the abstract the values from the "data fit" are given.

Table 2 shows the 3 temperatures, the emission measures in $10^{51} \mathrm{~cm}^{-3}$, and abundances together with their standard deviations within parentheses. The abundances are normalized to their corresponding solar photospheric values (Anders \& Grevesse 1989), except for iron and oxygen. For $\mathrm{Fe}$ we have changed the value of $\log A_{\mathrm{Fe}}$ from 7.67 (Anders \& Grevesse 1989) to 7.51 (Grevesse \& Sauval 1998, 1999). For oxygen we used the new value given by

\footnotetext{
${ }^{1}$ http://www.sron.nl/divisions/hea/spex/version1.10/ line/line_new.ps.gz
} 

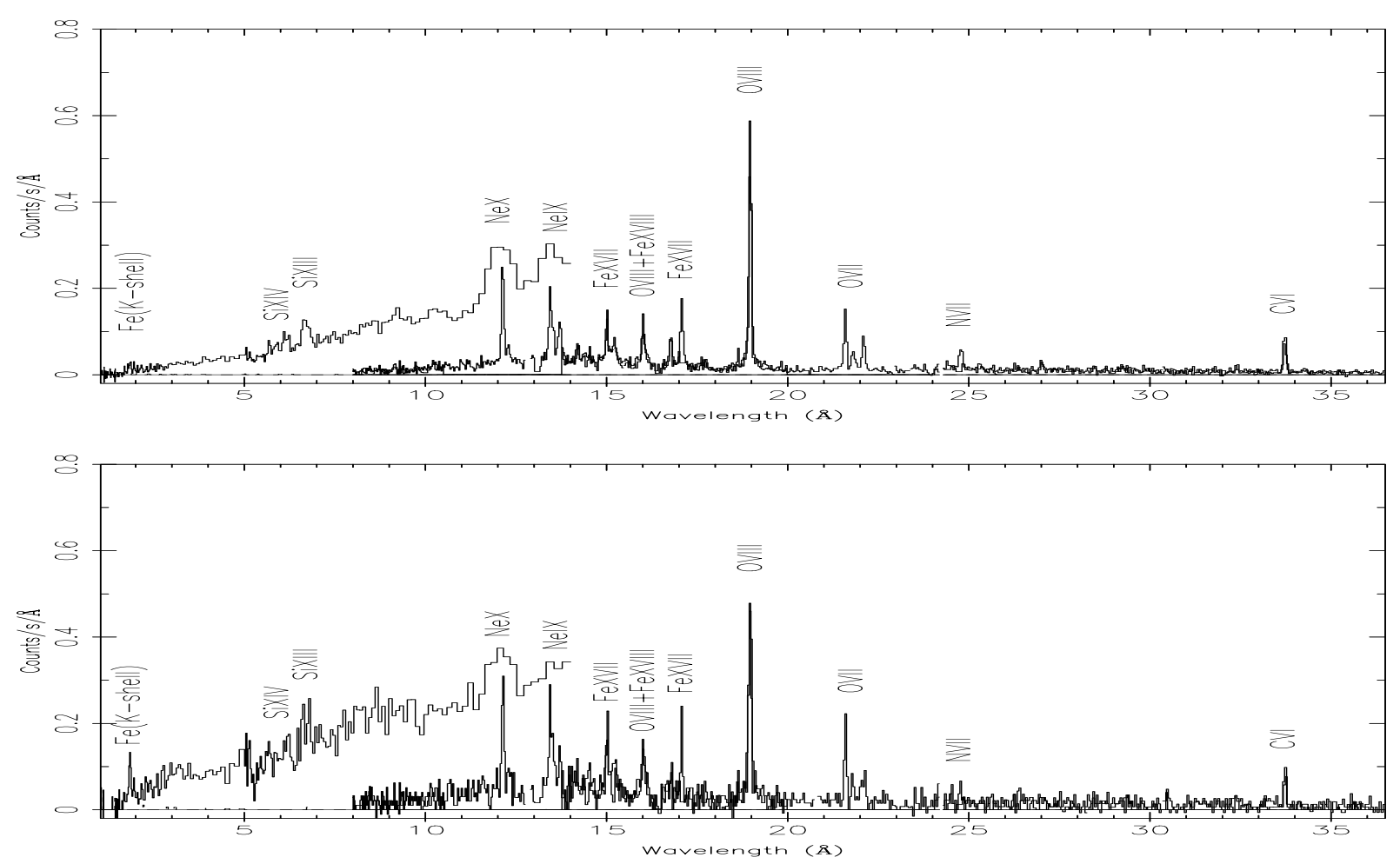

Fig. 2. The spectra of AT Mic observed by RGS1, RGS2 and EPIC-MOS in the wavelength region from 1 to $37 \AA$. The upper panel shows the quiescent state, while the lower panel shows the flaring state.

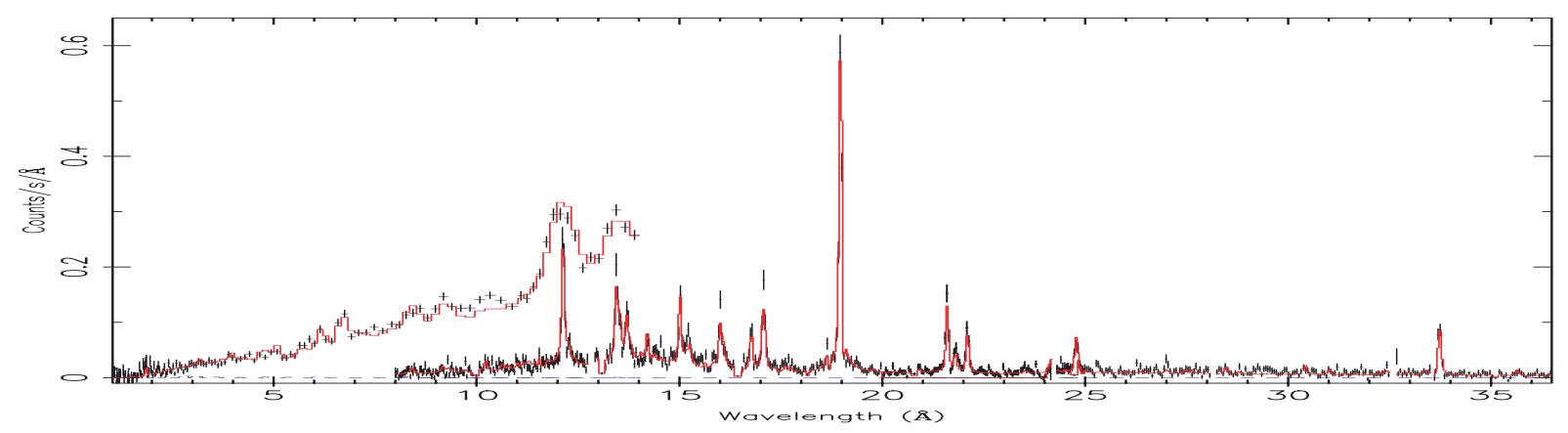

Fig. 3. Comparison of the AT Mic quiescent spectrum (see Fig. 2a) with the best-fit model (unbroken line).

Allende Prieto \& Lambert (2001) $\left(\log A_{\mathrm{O}}=8.69\right.$ instead of 8.93). Here $\log A_{\mathrm{Fe}}$ and $\log A_{\mathrm{O}}$ is the logarithm of the $\mathrm{Fe}$ - and O-abundance relative to $\log A_{\mathrm{H}}=12.0$.

The temperatures in Table 2 cover a range from 3 to $35 \mathrm{MK}$. It turns out that the line spectrum is mainly produced by the $E M$ related to $T_{1}=3 \mathrm{MK}$ and to $T_{2}=8 \mathrm{MK}$, while the hot component $T_{3}>20 \mathrm{MK}$ contributes to the continuum below $10 \AA$ and the Fe K-shell lines in the EPIC-MOS observation. This is especially true for the flaring state. A fourth cool component $(1 \mathrm{MK})$ is possible but not well constrained. This cool component is only related to the $\mathrm{C}$ VI line (influencing the $\mathrm{C}$ abundance) and very weakly coupled to the continuum. The total emission measure of the three components is $12.2(.5) \times$ $10^{51} \mathrm{~cm}^{-3}$ for the quiescent state and $19.5(.8) \times 10^{51} \mathrm{~cm}^{-3}$ for the flare state. Based on EUVE observations, Monsignori Fossi et al. (1995) have determined an emission measure maximum of about $3 \times 10^{51} \mathrm{~cm}^{-3}$ around $10 \mathrm{MK}$ (their Fig. 5), detecting only the emission measure related to our second temperature component at $T_{2}(7.9 \mathrm{MK})$.

When fitting the O VII lines a slight increase of the electron density $n_{\mathrm{e}}$ is noticed, from the quiescent state to the flaring state. However, this increase is not statistically significant.

In the energy interval from 0.3 to $10 \mathrm{keV}$ the $\mathrm{X}$-ray luminoscity $L_{\mathrm{x}}$ is $2.13(.08) \times 10^{29} \mathrm{erg} / \mathrm{s}$ for the quiescent state and $3.63(.22) \times 10^{29} \mathrm{erg} / \mathrm{s}$ for the flaring state. These values are averages of the "data" and "model" fit. Pallavicini et al. (1990) observed AT Mic with EXOSAT (ME 1-10 keV detector) during quiescence and during a flare. The quiescent X-ray luminosity was measured to be $1.8 \times 10^{29} \mathrm{erg} / \mathrm{s}$ (Table 5 of Pallavicini et al. 1990) in agreement with our value. However, they observed a larger flare, but with the same temperature as ours. 
Table 2. Multi-temperature fitting to the quiescent and flaring state of AT Mic; $1 \sigma$ uncertainties are given in the last digits in parentheses.

\begin{tabular}{|c|c|c|c|c|}
\hline \multirow[t]{2}{*}{ PARAMETER } & \multicolumn{2}{|c|}{ QUIESCENT $^{a}$} & \multicolumn{2}{|c|}{ FLARING } \\
\hline & data $^{b}$ & model $^{b}$ & data & model \\
\hline $\log N_{\mathrm{H}}\left[\mathrm{cm}^{-2}\right]$ & $18.3^{c}$ & $18.3^{c}$ & $18.3^{c}$ & $18.3^{c}$ \\
\hline$T_{1}[\mathrm{MK}]$ & $3.16(.10)$ & $2.94(.10)$ & $2.91(.17)$ & $2.71(.20)$ \\
\hline$T_{2}[\mathrm{MK}]$ & $7.77(.13)$ & $7.54(.14)$ & $8.57(.34)$ & $7.80(.27)$ \\
\hline$T_{3}[\mathrm{MK}]$ & $23.0(1.0)$ & $27.9(2.3)$ & $33.9(3.2)$ & $32.8(5.1)$ \\
\hline$E M_{1}\left[10^{51} \mathrm{~cm}^{-3}\right]$ & $2.34(.16)$ & $3.86(.41)$ & $2.71(.31)$ & $4.95(1.3)$ \\
\hline$E M_{2}\left[10^{51} \mathrm{~cm}^{-3}\right]$ & $4.85(.26)$ & $8.58(.93)$ & $4.27(.42)$ & $10.9(4.0)$ \\
\hline$E M_{3}\left[10^{51} \mathrm{~cm}^{-3}\right]$ & $4.99(.20)$ & $5.51(.44)$ & $12.47(.61)$ & $16.5(1.9)$ \\
\hline$E M_{\text {total }}\left[10^{51} \mathrm{~cm}^{-3}\right]$ & $12.2(.5)$ & $17.8(1.1)$ & $19.5(.8)$ & $32.4(4.6)$ \\
\hline$n_{\mathrm{e}}(\mathrm{O})\left[10^{10} \mathrm{~cm}^{-3}\right]^{d}$ & $1.9(1.5)$ & $1.4(1.4)$ & $4(-3,+5)$ & $3(-3,+4)$ \\
\hline$L_{\mathrm{x}}\left[10^{29} \mathrm{erg} / \mathrm{s}\right]^{e}$ & $2.05(.08)$ & $2.26(.14)$ & $3.54(.15)$ & $3.98(.57)$ \\
\hline $\mathrm{Mg} / \mathrm{O} 7.65 \mathrm{eV}$ & $0.51(.06)$ & $0.44(.09)$ & $1.00(.22)$ & $0.68(.28)$ \\
\hline $\mathrm{Fe} / \mathrm{O} \quad 7.87 \mathrm{eV}$ & $0.34(.02)$ & $0.31(.05)$ & $0.56(.04)$ & $0.45(.16)$ \\
\hline $\mathrm{Si} / \mathrm{O} \quad 8.15 \mathrm{eV}$ & $0.60(.06)$ & $0.69(.11)$ & $0.72(.22)$ & $0.68(28)$ \\
\hline $\mathrm{C} / \mathrm{O} \quad 11.26 \mathrm{eV}$ & $1.00(.10)$ & $1.01(.19)$ & $0.83(.21)$ & $0.84(.30)$ \\
\hline $\mathrm{O} / \mathrm{O} \quad 13.62 \mathrm{eV}$ & $1.00(.05)$ & $1.00(.14)$ & $1.00(.10)$ & $1.00(.25)$ \\
\hline $\mathrm{N} / \mathrm{O} \quad 14.53 \mathrm{eV}$ & $0.86(.09)$ & $0.73(.16)$ & $0.58(.27)$ & $0.51(.31)$ \\
\hline $\mathrm{Ne} / \mathrm{O} \quad 21.56 \mathrm{eV}$ & $1.52(.08)$ & $1.48(.20)$ & $1.90(.21)$ & $1.50(.49)$ \\
\hline $\mathrm{O} / \mathrm{H} \quad 13.62 \mathrm{eV}$ & $1.57(.05)$ & $0.96(.09)$ & $1.43(.10)$ & $0.71(.13)$ \\
\hline$E M_{\text {total }} \times \mathrm{O} / \mathrm{H}$ & 19.2(1.0) & $17.1(2.0)$ & $27.9(2.3)$ & $23.0(5.3)$ \\
\hline$\chi^{2} /$ d.o.f. & 1.1 & 1.4 & 0.60 & 1.10 \\
\hline
\end{tabular}

${ }^{a}$ Based on good time interval (with low solar proton flux) and excluding the stellar flare (see Fig. 5).

${ }^{b}$ Data stands for "fit weight data" and model stands for "fit weight model".

${ }^{c}$ Fixed at literature value from Monsignori Fossi et al. (1995).

${ }^{d}$ Based on O VII lines only.

${ }^{e}$ In the $0.3-10 \mathrm{keV}$ energy interval.

The abundances have been determined by fitting to the spectrum as a whole. As a check, whether a large scatter exists between different lines of the same element, abundances were also fitted to isolated individual lines, keeping temperatures, emission measures and other abundances fixed at the values from the global fit (Table 2). In this way blends have been taken into account. Due to the weak continuum the absolute abundances cannot be coupled accurately to the continuum and therefore they are strongly anticorrelated with the emission measure. This means that if one quantity increases the other decreases and vice versa. This is clear from comparing the $E M_{\text {total }}$ from the "data fit" with that from the "model fit" and by comparing the absolute oxygen abundance $(\mathrm{O} / \mathrm{H})$ in these columns. The product $E M_{\text {total }} \times \mathrm{O} / \mathrm{H}$, however, is more stable. For this reason the abundance ratios, normalized to oxygen, are more robust. Oxygen was chosen for its low relative standard deviation, thanks to the strong O VIII and O VII lines. For the (less active) solar corona Feldman et al. (1992) discussed the presence of a First Ionization Potential (FIP) effect. This effect implies that elements with a low value of the first ionization potential (e.g., $\lesssim 10 \mathrm{eV}$ ) show enhanced coronal abundances relative
Table 3. Ratio between the scaled abundance values of the flaring and quiescent state from Table 2.

\begin{tabular}{lll}
\hline \hline \multicolumn{2}{l}{ Parameter } & flaring/quiescent \\
\hline $\mathrm{Mg} / \mathrm{O}$ & $7.65 \mathrm{eV}$ & $1.75(.42)$ \\
$\mathrm{Fe} / \mathrm{O}$ & $7.87 \mathrm{eV}$ & $1.63(.20)$ \\
$\mathrm{Si} / \mathrm{O}$ & $8.15 \mathrm{eV}$ & $1.11(.30)$ \\
$\mathrm{C} / \mathrm{O}$ & $11.26 \mathrm{eV}$ & $0.83(.20)$ \\
$\mathrm{O} / \mathrm{O}$ & $13.62 \mathrm{eV}$ & $1.00(.11)$ \\
$\mathrm{N} / \mathrm{O}$ & $14.53 \mathrm{eV}$ & $0.68(.27)$ \\
$\mathrm{Ne} / \mathrm{O}$ & $21.56 \mathrm{eV}$ & $1.19(.17)$ \\
\hline
\end{tabular}

to photospheric values. However, the abundance ratios of the low-FIP elements $\mathrm{Mg}, \mathrm{Si}$, and $\mathrm{Fe}$ are relatively low in AT Mic, indicating an inverse FIP-effect (IFIP). Similar IFIP effects were observed by Brinkman et al. (2001), Güdel et al. (2001), Audard et al. (2002) and Audard et al. (2003) for other active late-type stars. This IFIP effect is illustrated in Fig. 4 (top), which shows the trend of an increasing abundance ratio with increasing FIP for the quiescent state. In the bottom panel the same quantities are shown for the flaring state. However, due to the short time interval all quantities determined in the flare spectrum have large statistical uncertainties. The poor signalto-noise ratio of the flaring state data is also reflected in the "too good" $\chi^{2} /$ d.o.f. of the multitemperature fit. This suggests an overestimate for the errors in this low count rate spectrum. For that reason the fits have also been performed, attaching the errors to the model, resulting in reasonable $\chi^{2} /$ d.o.f.-values.

Comparing the quiescent state with the flaring state some flattening of this IFIP effect for the low FIP elements (Fe and $\mathrm{Mg}$ ) is noticed in the flaring state. This is shown in Table 3 and Fig. 4c, in which the ratios between the $\mathrm{A} / \mathrm{O}$ values of the flaring and quiescent state are given. The values used in Table 3 and Fig. 4 are averages between "data" and "model" values. Si seems not to be affected by the difference between the flaring state and the quiescent state. In the solar corona the enhancement of the low-FIP elements starts from $10 \mathrm{eV}$ down. Results in Table 3 and Fig. 4c may indicate a value lower than $10 \mathrm{eV}$ below which the elemental abundances are influenced. Such a shift might be related to the lower surface temperature of AT Mic.

\subsection{DEM-modeling}

To show the smooth connection between the three separated temperature components of the multi-temperature fit (Table 2) we have performed a DEM-modeling of the RGS and EPIC-MOS spectra of AT Mic in the quiescent and flaring state, applying the regularization method and the polynomial method present in the SPEX-code (see Kaastra et al. 1996b). In this DEMmodeling the abundance ratios given in Table 2 have been used in combination with an $\mathrm{O} / \mathrm{H}$ value of 1.57 for the quiescent state and 1.39 for the flare state. The emission measures $E M=n_{\mathrm{e}} n_{\mathrm{H}} V$ (per logarithmic temperature bin) of the corona of AT Mic are shown in Figs. 5a,b for both states. The emission measure is distributed from $\sim 1$ up to about $60 \mathrm{MK}$ for the 

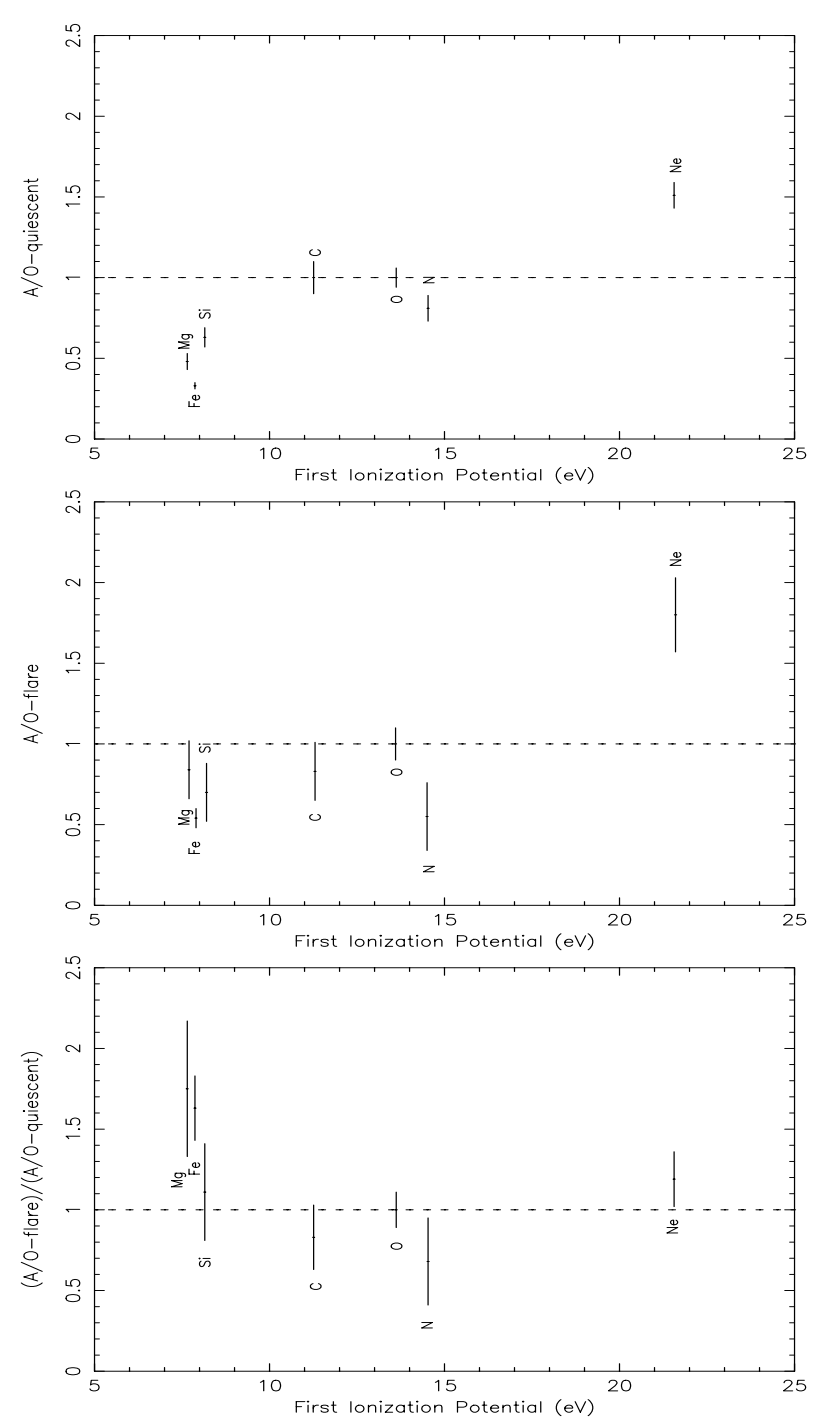

Fig. 4. Abundance ratio (A/O) versus First Ionization Potential, relative to solar photospheric values by Anders \& Grevesse (1989) and Grevesse \& Sauval (1998 and 1999) and with oxygen values from Allende Prieto \& Lambert (2001) for the quiescent state (top) and the flaring state (middle). The bottom panel shows the ratio between the $\mathrm{A} / \mathrm{O}$ of the flaring state and the $\mathrm{A} / \mathrm{O}$ of the quiescent state (Table 3 ).

flare state. The DEM distribution agrees with the three temperature components from the multi-temperature fit if we take into account the uncertainties corresponding to the standard deviations.

Table 2 and Fig. 5 show the increase of the emission measure and a shift to higher temperatures for the hot $E M$ (40 MK) during the flare state (bold) compared to the quiescent state (weak). The emission measure below $10 \mathrm{MK}$ is the same for the quiescent and flaring state.

\subsection{Individual lines}

The more prominent lines of the RGS spectrum have been measured individually. Delta functions were folded through the instrumental response matrix in order to derive integrated line fluxes. No additional width to the delta functions was needed
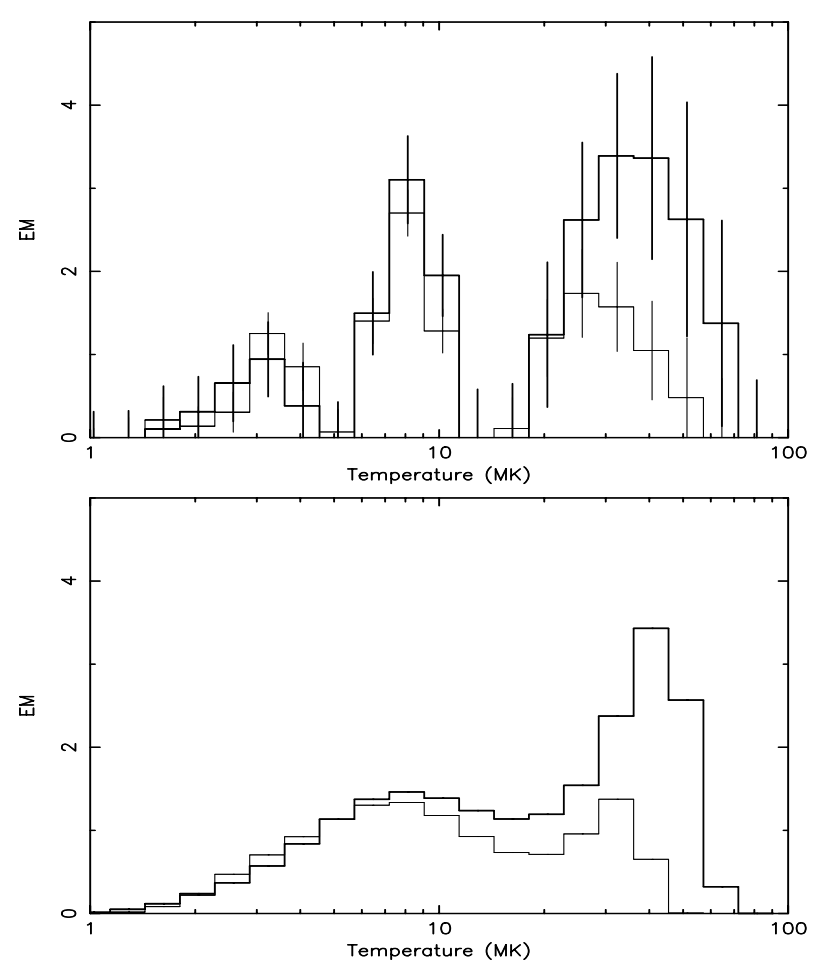

Fig. 5. DEM modeling of the flare spectrum (bold) and quiescent spectrum (weak) of AT Mic in units $10^{51} \mathrm{~cm}^{-3}$, using the regularization method (top panel). The bottom panel shows the same for the polynomial method of order 9 .

to fit the line shapes. A "constant" level was adjusted to take into account the real continuum or the "pseudo-continuum", created by the overlap of numerous weak lines.

The line fluxes are compared between the quiescent and flaring state (Table 4). Especially the flux difference of the Fe XXV line at $1.84 \AA$ between the two states is clear. This line has a far higher flux in the flare state than in the quiescent state. The same is true for other highly ionized (hot) ions, such as Si XIV, Si XIII, Fe XIX, Fe XVIII, and Fe XVII. However, for these ions the effect is less significant.

\subsection{Density measurement}

The three He-like transitions, consisting of the resonance line (r) $1 \mathrm{~s}^{2}{ }^{1} \mathrm{~S}_{0}-1 \mathrm{~s} 2 \mathrm{p}{ }^{1} \mathrm{P}_{1}$, the intercombination line (i) $1 \mathrm{~s}^{2}{ }^{1} \mathrm{~S}_{0}-1 \mathrm{~s} 2 \mathrm{p}{ }^{3} \mathrm{P}_{1}$, and the forbidden line (f) $1 \mathrm{~s}^{2}{ }^{1} \mathrm{~S}_{0^{-}}$ $1 \mathrm{~s} 2 \mathrm{~s}{ }^{3} \mathrm{~S}_{1}$ are density- and temperature-dependent (e.g., Gabriel \& Jordan 1969). At increasing electron density the lowlying $1 \mathrm{~s} 2 \mathrm{~s}{ }^{3} \mathrm{~S}_{1}$ level will be depopulated in favour of the $1 \mathrm{~s} 2 \mathrm{p}{ }^{3} \mathrm{P}_{1}$ level, resulting in a decrease of the intensity of the forbidden line and an increase of the intercombination line. This makes the ratio $R=f / i$ a valuable diagnostic tool for densities. The He-like lines of oxygen are well separated, although the intercombination line in the quiescent spectrum is affected by an innershell transition in O VI (see Fig. 6). These lines at $21.602 \AA(r), 21.802 \AA(i)$ and $22.101 \AA(f)$ have been used to obtain temperature and density values from the $G$ - and $R$-ratio using the tables by Porquet et al. (2001). For representative temperatures around $3 \mathrm{MK}$, we derive electron densities of 
Table 4. Observed line fluxes of the quiescent and flare spectra of AT Mic.

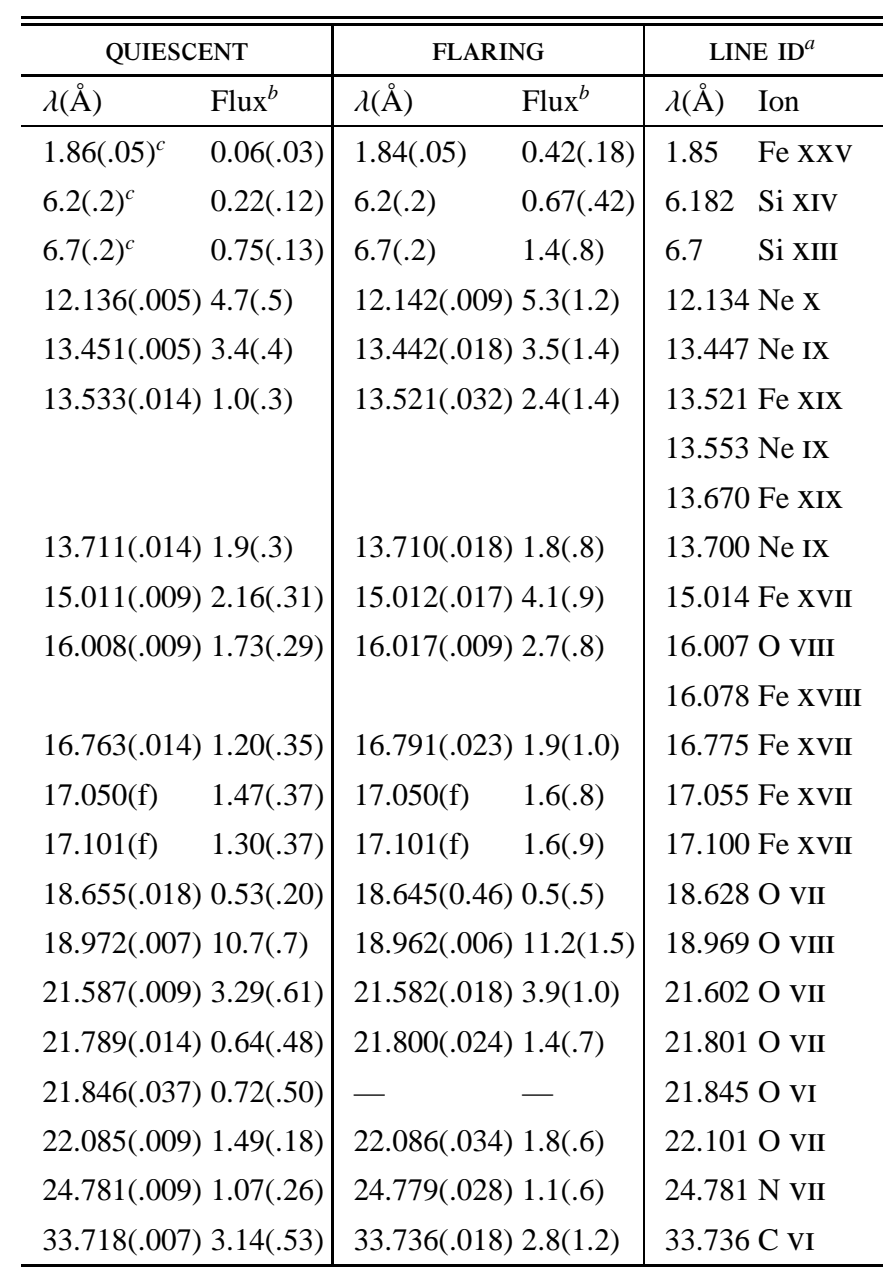

${ }^{a}$ Line identification from Kelly (1987).

${ }^{b}$ Measured flux in $10^{-4}$ photons $/ \mathrm{cm}^{2} / \mathrm{s}$.

${ }^{c}$ Wavelengths and fluxes from EPIC-MOS.

$3 \pm 2 \times 10^{10} \mathrm{~cm}^{-3}$ for the quiescent state and $8_{-7.5}^{+20} \times 10^{10} \mathrm{~cm}^{-3}$ for the flaring state. Within the errors these values agree with those given in Table 2 .

\section{Conclusions}

The X-ray spectrum of AT Mic obtained by RGS and EPIC-MOS is dominated by $\mathrm{H}$ - and He-like transitions of $\mathrm{Ne}, \mathrm{O}, \mathrm{N}$, and $\mathrm{C}$ and by Fe XVII lines (RGS) and by $\mathrm{H}$ - and He-like transitions of $\mathrm{Si}$ and $\mathrm{Mg}$ and $\mathrm{Fe} \mathrm{K}$-shell transitions (EPIC-MOS).

The spectra correspond to a quiescent and a flaring state as was concluded from the observed light curve. The dominant temperatures and emission measures cover a broad temperature range from 1 up to about $60 \mathrm{MK}$. The total emission measure in this temperature domain is $\sim 12.2 \times 10^{51} \mathrm{~cm}^{-3}$ for the quiescent state and $\sim 19.5 \times 10^{51} \mathrm{~cm}^{-3}$ for the flaring state. Both values are obtained from the "fit weight data" fits.

From the $\mathrm{O}$ VII lines a density of $\sim 2 \times 10^{10} \mathrm{~cm}^{-3}$ is derived for the quiescent state and of $\sim 4 \times 10^{10} \mathrm{~cm}^{-3}$ for the flaring
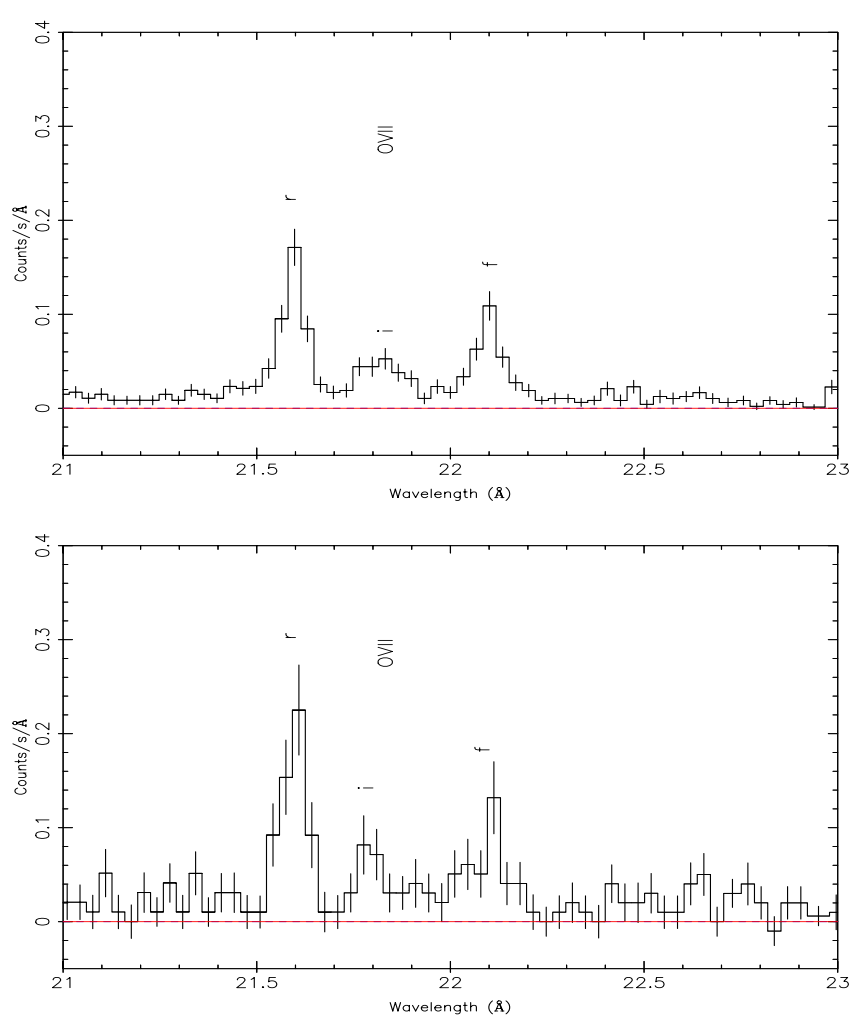

Fig. 6. The O VII lines of AT Mic observed by RGS1. The top panel shows the quiescent state, while the lower panel shows the flaring state.

state. The density of the flaring state is somewhat higher, but due to the poor statistics this is not significant.

Most line features of the RGS spectra are produced in the temperature range from 3-10 MK, dominated by quiescent emission. Lines from highly ionized atoms are enhanced in the flare state spectrum.

The abundance pattern in AT Mic shows the depletion of low-FIP elements $(<10 \mathrm{eV})$ relative to high-FIP elements in the quiescent state. This indicates the presence of a possible IFIP effect in this active star, as has been shown for other active stars by Audard et al. (2002), Audard et al. (2003), Brinkman et al. (2001), Güdel et al. (2001), and Güdel et al. (2002). However, in the flare state this effect appears to be suppressed. This may imply that during the flare material from deeper layers with photosperic/chromospheric abundances is transported into the corona.

Acknowledgements. The SRON National Institute for Space Research is supported financially by NWO. The PSI group acknowledges support from the Swiss National Science Foundation (grant 2100049343). MA acknowledges support from the Swiss National Science Foundation (fellowship 81EZ-67388).

\section{References}

Allende Prieto, C., \& Lambert, D. L. 2001, ApJ, 556, L63

Anders, E., \& Grevesse, N. 1989, Geochim. Cosmochim. Acta, 53, 197 
Audard, M., Güdel, M., Sres, A., et al. 2002, in Stellar Coronae in the Chandra and XMM-Newton Era, ed. F. Favata, \& J. J. Drake, ASP Conf. Ser., in press

Audard, M., Güdel, M., Sres, A., Raassen, A. J. J., \& Mewe, R. 2003, A\&A, 398, 1137

Brinkman, A. C., Gunsing, C. J. T., Kaastra, J. S., et al. 2000, ApJ, 530, L111

Brinkman, A. C., Behar, E., Güdel, M., et al. 2001, A\&A, 365, L324

Canizares, C. R., Huenemoerder, D. P., Davis, D. S., et al. 2000, ApJ, 539, L41

Feldman, U., Mandelbaum, P., Seely, J. F., et al. 1992, ApJS, 81, 387

Gabriel, A. H., \& Jordan, C. 1969, MNRAS, 145, 241

García-Alvarez, D., Jevremović, D., Doyle, J. G., \& Butler, C. J. 2002, A\&A, 383, 548

Grevesse, N., \& Sauval, A. J. 1998, Space Sci. Rev., 85, 161

Grevesse, N., \& Sauval, A. J. 1999, A\&A, 347, 348

Güdel, M., Audard, M., Briggs, K., et al. 2001, A\&A, 365, L336

Güdel, M., Audard, M., Smith, K. W., et al. 2002, ASP Conf. Ser., Cool Stars workshop, 12

den Herder, J. W., Brinkman, A. C., Kahn, S. M., et al. 2001, A\&A, 365, L7
Joy, A. H., \& Wilson, R. E. 1949, ApJ, 109, 231

Kaastra, J. S., Mewe, R., \& Nieuwenhuijzen, H. 1996a, in UV and X-ray Spectroscopy of Astrophysical and Laboratory Plasmas, ed. K. Yamashita, \& T. Watanabe (Tokyo: Universal Academy Press, Inc.), 411 (SPEX)

Kaastra, J. S., Mewe, R., Liedahl, D. A., et al. 1996b, A\&A, 314, 547 Kahn, S. M., Linsky, J. L., Mason, K. O., et al. 1979, ApJ, 234, L107 Mewe, R. 1991, A\&ARv, 3, 127

Mewe, R., Kaastra, J. S., \& Liedahl, D. A. 1995, Legacy, 6, 16 (MEKAL)

Monsignori Fossi, B. C., Landini, M., \& Drake, J. J. 1995, A\&A, 302, 193

Pallavicini, R. 1989, A\&ARv, 1, 177

Pallavicini, R., Tagliaferri, G., \& Stella, L. 1990, A\&A, 228, 403

Perryman, M. A. C., Lindegren, L., Kovalevsky, J., et al. 1997, A\&A, 323, L49

Pettersen, B. R. 1989, Sol. Phys., 121, 299

Phillips, K. J. H., Mewe, R., Harra-Murnion, L. K., et al. 1999, A\&AS, 138,381

Porquet, D., Mewe, R., Dubau. J., et al. 2001, A\&A, 376, 1113

Turner, M. J. L., Abbey, A., Arnaud, M., et al. 2001, A\&A, 365, L27 